\title{
Pedestrian risk perception in signalized street crossings
}

\author{
N. Eliou, A. Galanis \& A. Proios \\ Department of Civil Engineering, University of Thessaly, Greece
}

\begin{abstract}
This paper examines the pedestrian perception of risk and behaviour in signalized street crossings with a flashing green beacon, while, simultaneously, the cars turn right or left with a flashing orange beacon. The pedestrians have right of way in situations like that. However, it is not always implemented by the drivers. As a result, the risk of the pedestrian crossing is raised, as they are forced to cross the street even in the red signalized phase.

This study took place in the Greek city of Volos in December 2008. 2024 pedestrians were examined with the use of a video camera in two signalized intersections in the center of the city. During the elaboration of the data, the pedestrians were separated by sex, age and, while crossing the street, whether they were alone or in a group.

The study presents the pedestrian behaviour and perception of risk as they wait to cross the street, either on the sidewalk or in the street and the choice of crossing the street either with a green or red light. It also, examines the cases where the drivers stopped to give right of way to the pedestrians.
\end{abstract}

Keywords: pedestrian risk perception, pedestrian safety, pedestrians' behaviour, drivers' behaviour, signalized crossing.

\section{Introduction}

Pedestrian risk perception and behaviour of crossing the street is a critical issue for their road safety. Pedestrian crosswalks are either signalized or not. Pedestrians do not always cross with the "walk" signal, but they cross the street many times when they consider these is a gap in the flow of the cars. This behaviour raises the risk, as they are vulnerable road users. 
Apart from the two types of the signalized pedestrian phase (walk, do not walk), there is another one. There is a type of intersection where the pedestrians cross the street with a flashing green beacon (walk carefully), while, simultaneously, the vehicles turn right or left with a flashing orange beacon. In this case, both the vehicles and the pedestrians mix their movement in the crosswalk and the interaction between drivers and pedestrians is more complex. In many cases, pedestrians do not have time to cross the street, forcing them selves to do so when they have a red signalized phase "do not walk signal".

The pedestrians' behaviour in crossing the street at crosswalks depends on many factors [1]. The pedestrians' sex, age and whether being alone or in a group play an important role in the decision of crossing the street and rejecting or accepting the traffic gap [2-4].

\section{Problem simulation}

\subsection{Objective}

Within this research we tried to examine the pedestrians' risk perception and behaviour in street crossings with a flashing green beacon, while simultaneously the cars turn right or left with a flashing orange beacon. The pedestrians always have right of way in situations like that. However, it is not always implemented by the drivers. As a result, the risk of the pedestrians' crossing the street is raised as they are forced to do so even in the red signalized phase (do not walk signal). The main questions of the study, were:

a) The pedestrians' behaviour in waiting crossing the street, either on the sidewalk or in the street.

b) The pedestrians' perception of risk with crossing the street either in a flashing green signalized phase or in a red signalized phase according to their characteristics.

c) The drivers' behaviour in giving right of way to the pedestrians when they cross the street at a flashing green signalized phase according to the pedestrians' characteristics.

\subsection{Investigation}

This study took place in the Greek city of Volos in December 2008. 2024 pedestrians were examined with the use of a video camera in two signalized intersections, between 11:00 - 13:00 in the morning and 18:00 - 20:00 in the evening. 917 pedestrians were examined in the first intersection and 1107 pedestrians in the second one. The first intersection is named "Dimitriados Kartali" and the second one "Iasonos - Kartali". Both the intersections are in the center of the city, close to each other, used by many vehicles and pedestrians (figure 1). The length of each examined crosswalk is $7 \mathrm{~m}$, equal to two vehicle lanes. In both intersections, the red signalized phase is $36 \mathrm{sec}$ and the flashing green signalized phase also $36 \mathrm{sec}$. 
During the elaboration of the data, the pedestrians were separated by the following parameters;

a) Sex (male, female)

b) Age (0-20, 20-40, 40-60 and over 60)

Using the data of the video camera, we examined each pedestrian's behaviour, waiting and crossing the street. Firstly, we examined if the pedestrians waited either on the sidewalk or in the street and if they waited alone or with other pedestrians. There were also some pedestrians that they did not wait at all, but moved ahead to the crosswalk. If pedestrians moved as a group, we considered them having the same behaviour.

Secondly, we examined the crossing of the street. We examined the drivers' behaviour, especially when they faced pedestrians in the crosswalk. The case of drivers' giving right of way in the crosswalks was examined only when the pedestrians crossed the street with a flashing green beacon and not with a red light, which was illegal and the vehicles legally had right of way. Moreover, we examined the pedestrians' behaviour when they crossed the street. We examined the pedestrians' choice of crossing either with a flashing green beacon or with a red light and when they did so, if they stopped in the crosswalk or not.

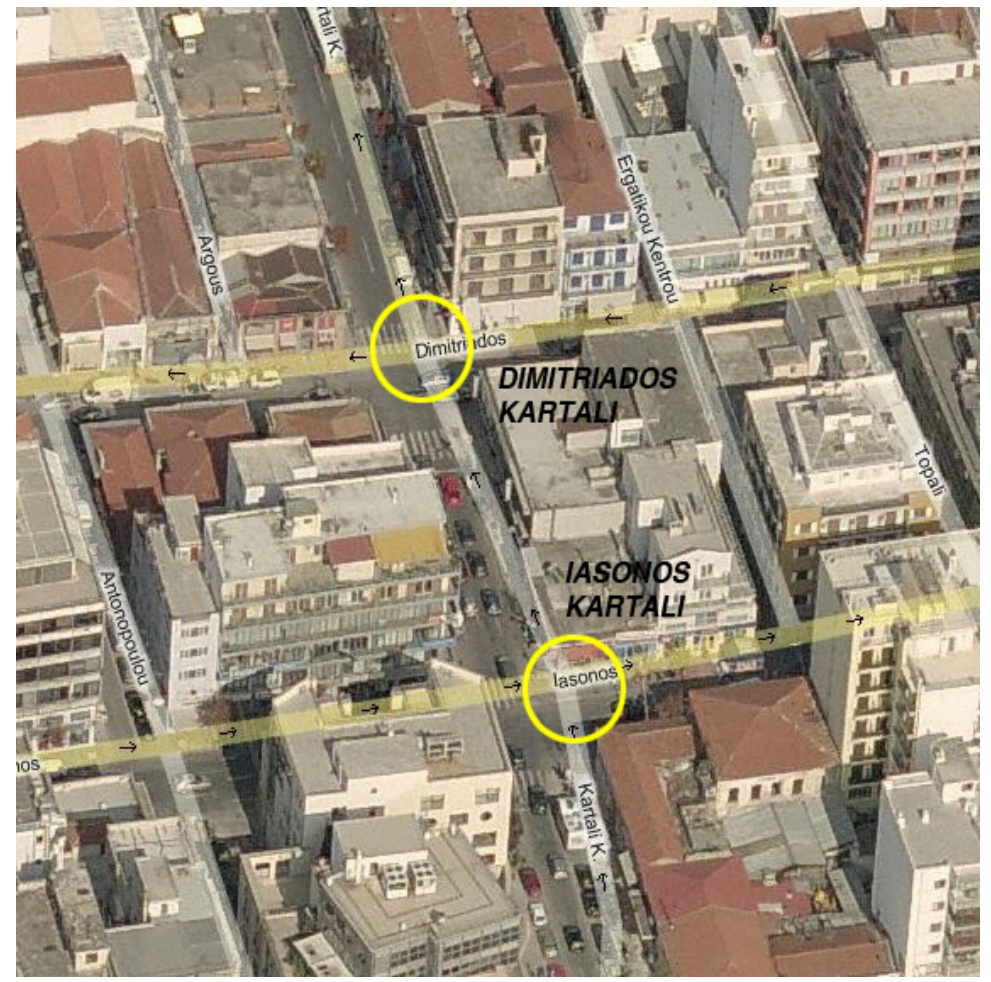

Figure 1: Intersections. 


\section{Results}

After watching the video of the first intersection "Dimitriados - Kartali", we classified the pedestrians during the crossing of the examined crosswalk. The duration of the video was $120 \mathrm{~min}, 75 \mathrm{~min}$ in the morning and $45 \mathrm{~min}$ in the afternoon. We counted 1107 pedestrians, 526 men $(47,5 \%)$ and 581 women (52,5\%), (figure 1). 129 pedestrians $(11,7 \%)$ were 0 -20 years old, $445(40,2 \%)$ were $20-40$ years old, $312(28,2 \%)$ were $40-60$ years old and $222(20,1 \%)$ were over 60 years old (figure 2). 446 of the pedestrians $(40,3 \%)$ crossed the street alone and $661(59,7 \%)$ in a group (figure 3). 294 pedestrians $(26,6 \%)$ crossed the street with a red light and $813(73,4 \%)$ with a green flashing beacon (figure 4$)$. 96 pedestrians $(11,8 \%)$ stopped on the street crossing with a flashing green beacon. 4 drivers $(8,2 \%)$ gave way to 12 pedestrians $(12,5 \%)$ and 45 drivers

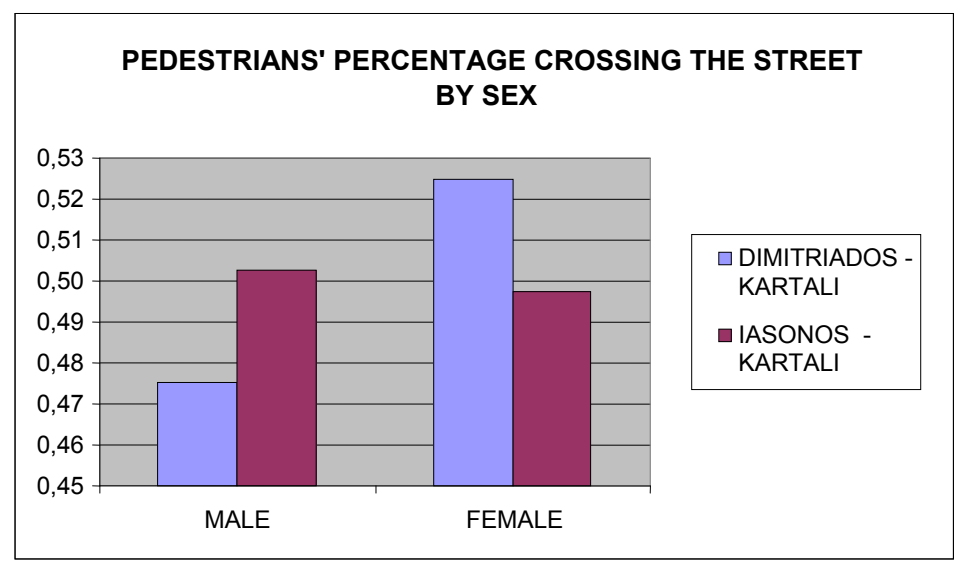

Figure 1: $\quad$ Pedestrians' percentage crossing the street by sex.

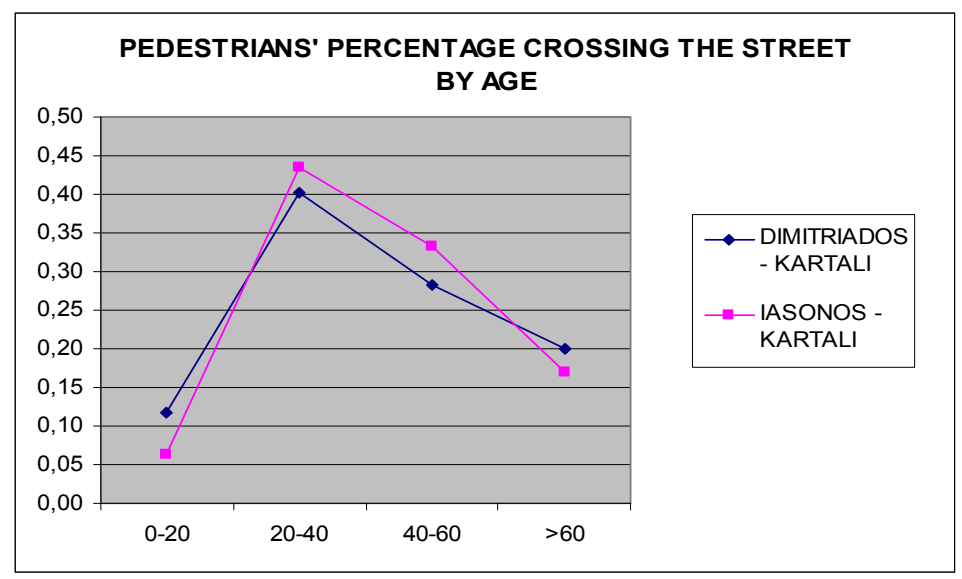

Figure 2: $\quad$ Pedestrians' percentage crossing the street by age. 


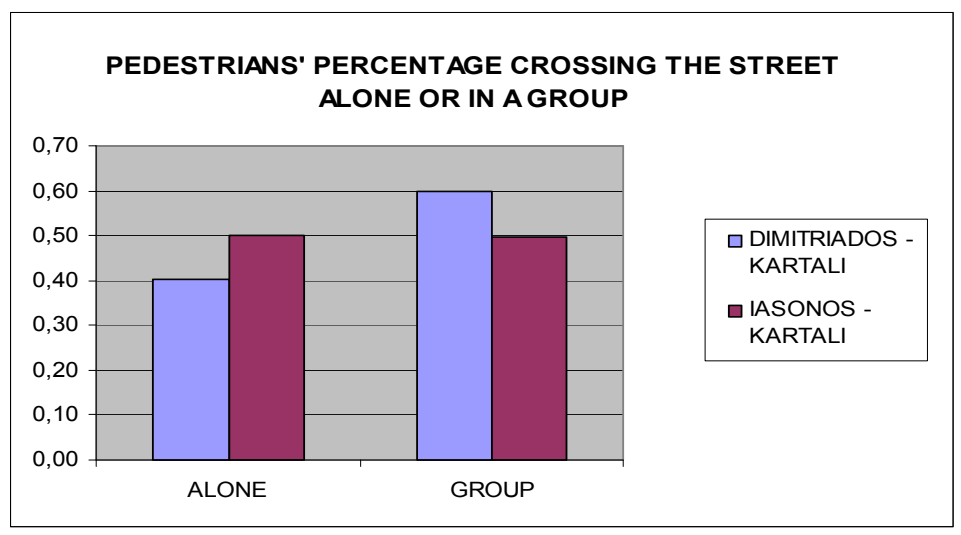

Figure 3: Pedestrians' percentage crossing the street alone or in a group.

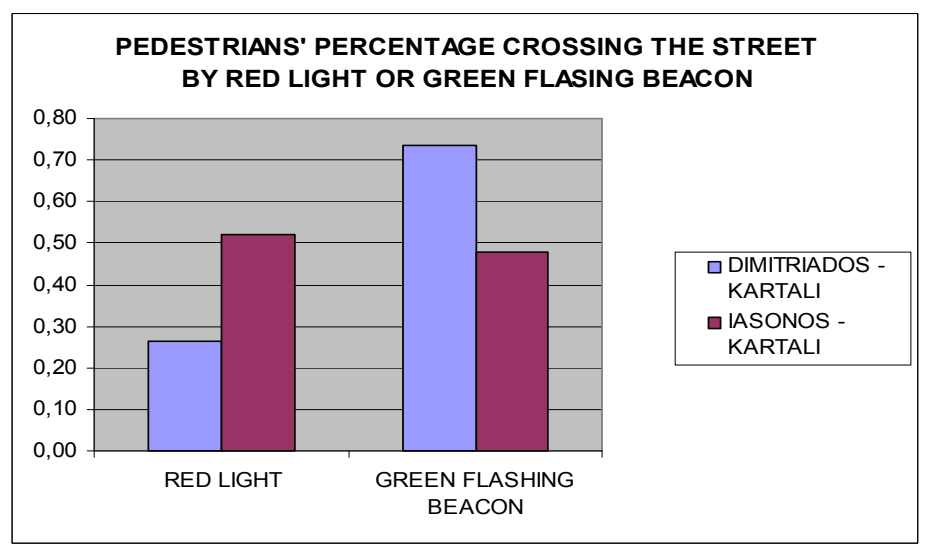

Figure 4: Pedestrians' percentage crossing the street by red light or green flashing beacon.

$(91,8 \%)$ did not give way to $84(87,5 \%) .560$ pedestrians $(50,6 \%)$ waited to cross the street and $547(49,4 \%)$ crossed the street without waiting. 353 pedestrians $(63 \%)$ waited on the sidewalk and $207(37 \%)$ waited in the street before crossing (figure 5). The average waiting time on the sidewalk was $6,66 \mathrm{sec}$ and in the street was $6,36 \mathrm{sec}$ (figure 6). The average total necessary time for crossing the street was $13,02 \mathrm{sec}$.

After watching the video of the second intersection "Iasonos - Kartali", we classified the pedestrians during the crossing of the examined crosswalk. The duration of the video was $180 \mathrm{~min}, 90 \mathrm{~min}$ in the morning and $90 \mathrm{~min}$ in the afternoon. We counted 917 pedestrians, 461 men $(50,3 \%)$ and 456 women $(49,7 \%)$, (figure 1). 58 pedestrians $(6,3 \%)$ were 0 -20 years old, $398(43,4 \%)$ were 20-40 years old, 305 (33,3\%) were 40-60 years old and $156(17 \%)$ were 
over 60 years old (figure 2). 460 of the pedestrians $(50,2 \%)$ crossed the street alone and $457(49,8 \%)$ in a group (figure 3$) .478$ pedestrians $(52,1 \%)$ crossed the street with a red light and 439 (47,9\%) with a flashing green beacon (figure 4). 113 pedestrians $(25,7 \%)$ stopped on the street crossing with a flashing green beacon. 7 drivers $(9,6 \%)$ gave way to 10 pedestrians $(8,8 \%)$ and 66 drivers $(90,4 \%)$ did not give way to $103(91,2 \%)$. 655 pedestrians $(71,7 \%)$ waited to cross the street and $262(28,3 \%)$ crossed the street without waiting. 445 pedestrians $(67,9 \%)$ waited on the sidewalk and $210(32,1 \%)$ waited in the street before crossing (figure 5). The average waiting time on the sidewalk was $6,37 \mathrm{sec}$ and in the street was $5,75 \mathrm{sec}$ (figure 6). The average total necessary time for crossing the street was $12,11 \mathrm{sec}$.

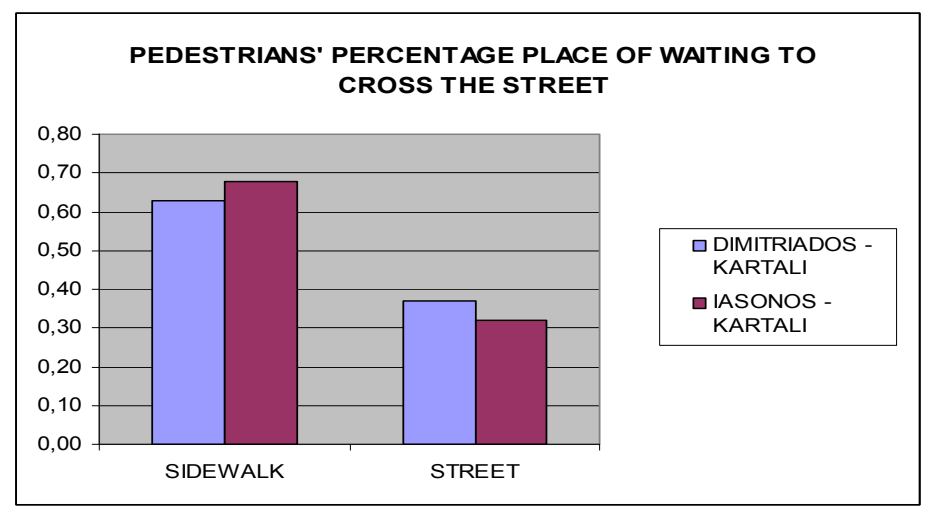

Figure 5: $\quad$ Pedestrians' percentage place of waiting to cross the street.

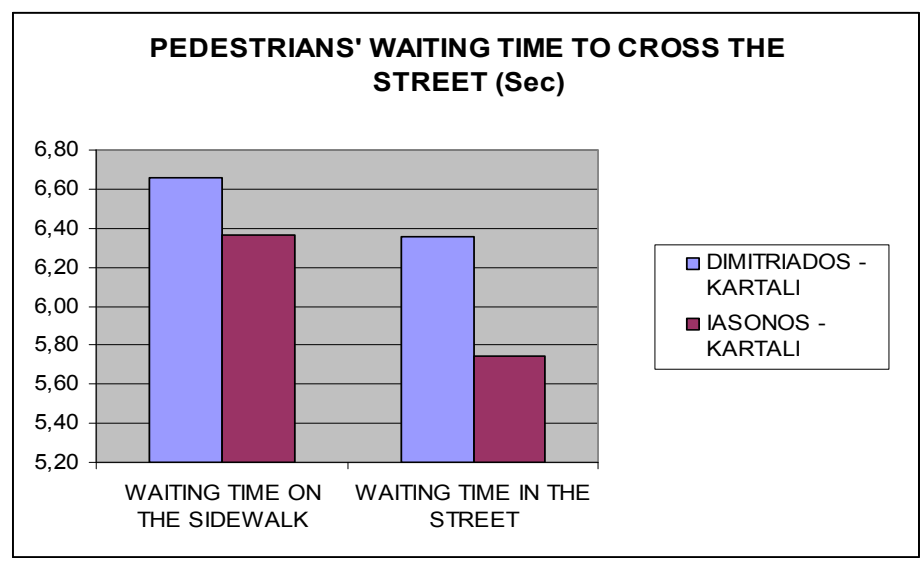

Figure 6: Pedestrians' waiting time to cross the street (sec).

We calculated the pedestrians' percentage, according to their age and sex, of crossing the street with a red light. In the first intersection "Dimitriados Kartali”, 147 men (27,9\%) and 147 women (25,3\%) crossed the street with a red 
light (figure 7). 33 pedestrians $(25,4 \%)$ 0-20 years old crossed the street with a red light, 113 pedestrians (25,5\%) 20-40 years old, $97(31,1 \%) 40-60$ years old and $51(23 \%)$ over 60 years old (figure 8). In the second intersection "Iasonos Kartali”, 249 men (54\%) and 229 women (50,2\%), (figure 7), crossed the street with a red light. 37 pedestrians $(63,8 \%)$ 0-20 years old crossed the street with a red light, 206 pedestrians (51,8\%) 20-40 years old, 155 (50,8\%) 40-60 years old and $80(51,3 \%)$ over 60 years old (figure 8$)$.

We calculated the pedestrians' percentage, according to their age and sex, of crossing the street with a flashing green beacon. In the first intersection "Dimitriados - Kartali", 379 men (72,1\%) and 434 women (74,7\%) crossed the street with a flashing green beacon. 97 pedestrians $(74,6 \%)$ 0-20 years old crossed the street with a red light, 330 pedestrians (74,5\%) 20-40 years old, 215 $(68,9 \%$ ) 40-60 years old and $171(77 \%)$ over 60 years old. In the second

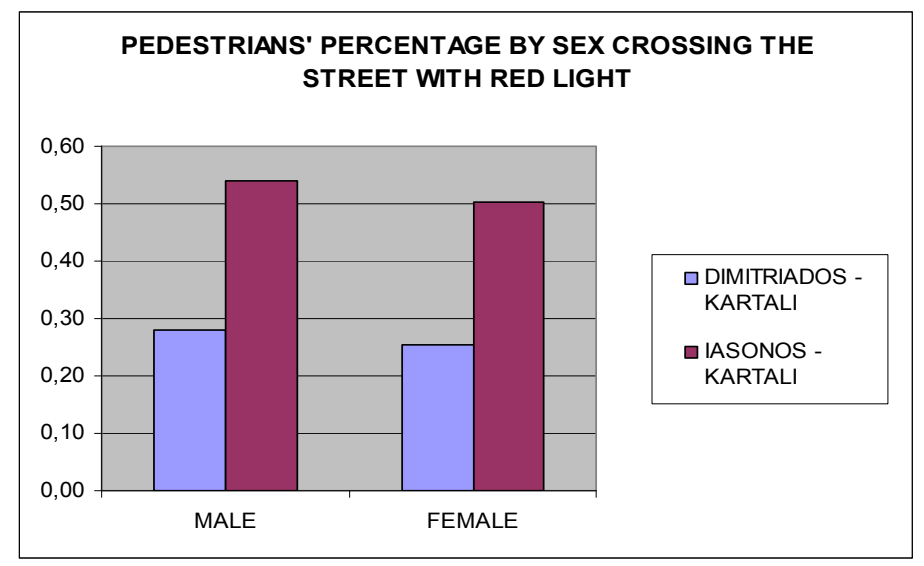

Figure 7: Pedestrians' percentage by sex crossing the street with red light.

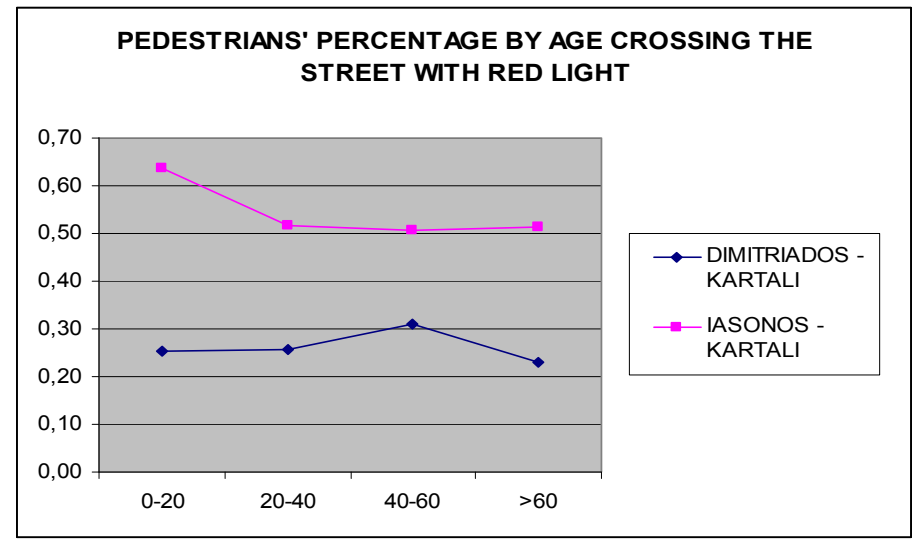

Figure 8: $\quad$ Pedestrians' percentage by age crossing the street with a red light. 


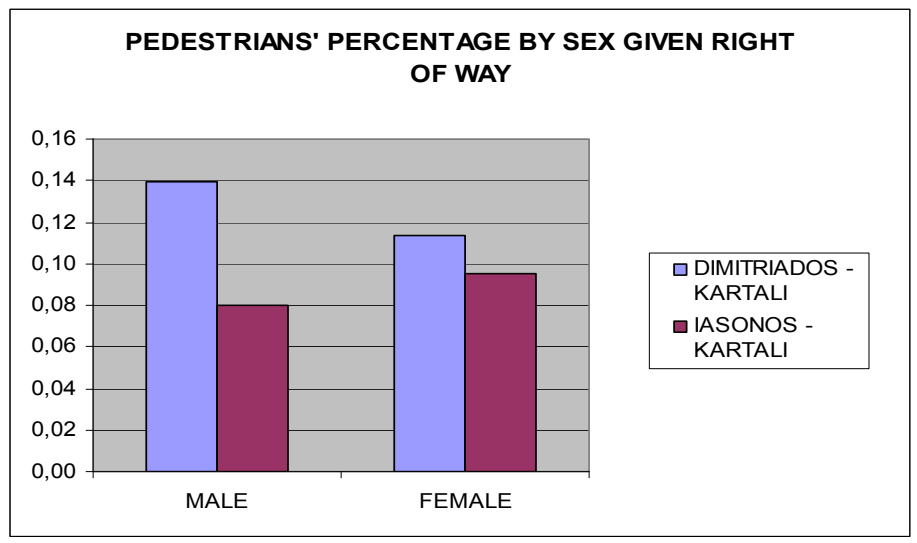

Figure 9: $\quad$ Pedestrians' percentage by sex given right of way.

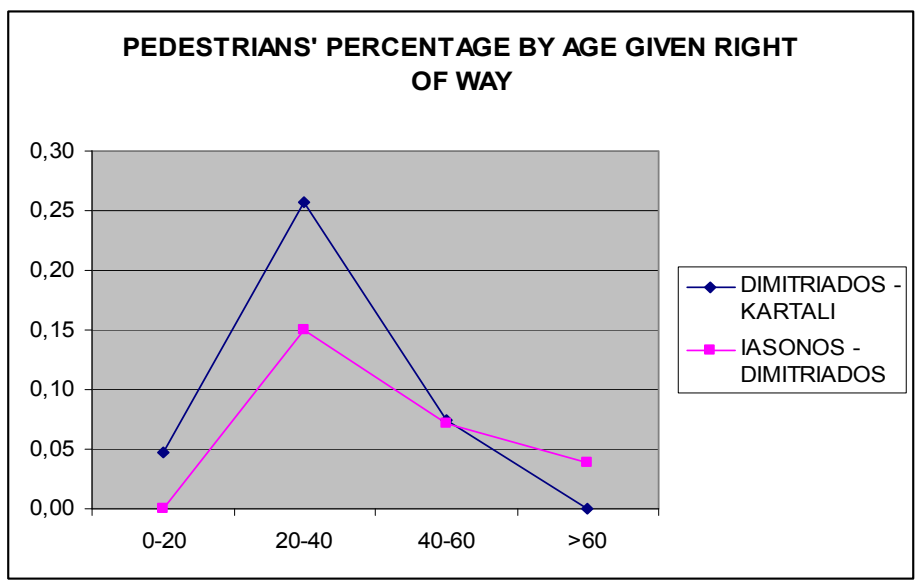

Figure 10: Pedestrians' percentage by age given right of way.

intersection "Iasonos - Kartali", 212 men (46\%) and 227 women (49,8\%), crossed the street with a flashing green beacon. 21 pedestrians $(36,2 \%)$ 0-20 years old crossed the street with a flashing green beacon, 192 pedestrians $(48,2 \%)$ 20-40 years old, $150(49,2 \%)$ 40-60 years old and $76(48,7 \%)$ over 60 years old.

We calculated the pedestrians' percentage given right of way by the drivers, according to the pedestrians' age, sex and being in a group or not, when they crossed the street with a flashing green beacon. In the first intersection, "Dimitriados - Kartali", 4 drivers gave right of way to 6 male pedestrians $(14 \%)$ and 6 female (11,3\%), (figure 9). 1 pedestrian was 0-20 years old $(4,8 \%), 9$ pedestrians were $20-40$ years old $(25,7 \%), 2$ pedestrians were $40-60$ years old $(7,4 \%)$ and 0 pedestrians were over 60 years old $(0 \%)$, (figure 10). 1 pedestrian crossed the street alone (4,5\%) and 11 in a group (14,9\%). In the second 
intersection "Iasonos - Kartali", 7 drivers gave right of way to 4 male pedestrians $(8 \%)$ and $6(9,5 \%)$, (figure 9). 0 pedestrians were 0 -20 years old $(0 \%), 6$ pedestrians were $20-40$ years old $(15 \%), 3$ pedestrians were $40-60$ years old $(7,1 \%)$ and 1 pedestrian was over 60 years old $(3,8 \%)$, (figure 10$)$. 4 pedestrians crossed the street alone $(6,9 \%)$ and 6 in a group $(10,9 \%)$.

\section{Comments}

After the elaboration of the results, we concluded on the followings:

a) About $35 \%$ of the pedestrians waited in the street and not on the sidewalk before they cross it, which raises the risk they take.

b) About $40 \%$ of the pedestrians cross the street with red light and $60 \%$ with flashing green beacon. Neither the sex nor the age influenced their choice.

c) About $10 \%$ of the drivers gave right of way to the pedestrians when they crossed the street with flashing green beacon and $90 \%$ did not. Pedestrians' sex does not influence this behaviour, but age does so. Drivers give right of way more often to pedestrians 20-40 years old.

This study shows the lack of proper driving culture of the Greek drivers and the lack of the pedestrians' ability to protect them selves even in signalized street crossings. Pedestrian take the risk to cross the street when they see a gap in the vehicle flow, even when there is a red light. It would be useful to examine both the pedestrians' and the drivers' characteristics of giving right of way to the pedestrians, in situations like that.

\section{References}

[1] Ariane Tom, Jean - Michel Auberlet and Roland Brebond, Perceptive and cognitive process in the pedestrian decision - making: How do pedestrians cross at intersections? Proceeding of the extra ICTCT - Workshop, 2007.

[2] T. Rosenbloom, Crossing at a red light: Behaviour of individuals and groups. Proceedings of the $4^{\text {th }}$ International Conference on Traffic \& Transport Psychology, 2008.

[3] C.A. Holland, Understanding the role of self-identity in road crossing decisions across the lifespan. Proceedings of the $4^{\text {th }}$ International Conference on Traffic \& Transport Psychology, 2008.

[4] A study on influence of gender and age on the gap acceptance behaviour of pedestrians in heterogeneous traffic situations. Proceedings of the $4^{\text {th }}$ International Conference on Traffic \& Transport Psychology, 2008. 